\title{
Efficacy of Paclitaxel in a Patient with Inoperable Pulmonary Vein Leiomyosarcoma
}

\author{
Shinji Kounami ${ }^{a}$ Hiroshi Tsujimoto $^{a}$ Takayuki Ichikawa $^{a}$ \\ Megumi Yoshiyamab ${ }^{b}$ Mitsuru Yuzaki $^{c}$ Yoshiharu Nishimura $^{c}$ \\ Hiroyuki Suzukia \\ ${ }^{a}$ Department of Pediatrics, Wakayama Medical University, Wakayama, Japan; ${ }^{b}$ Division of \\ Pediatrics, Hidaka General Hospital, Gobo, Japan; ${ }^{C}$ Department of Thoracic and \\ Cardiovascular Surgery, Wakayama Medical University, Wakayama, Japan
}

\section{Keywords}

Leiomyosarcoma · Pulmonary vein · Paclitaxel · High-dose chemotherapy · Peripheral blood stem cell transplantation

\begin{abstract}
Pulmonary vein leiomyosarcoma is extremely rare and has a poor prognosis. Surgical resection with a wide margin seems to offer the only chance of cure. The role of adjuvant therapy is controversial, and the exact efficacy of chemotherapy has not been observed. In this report, we present an 18-year-old male patient with pulmonary vein leiomyosarcoma in whom the use of paclitaxel (PAX) proved to be effective. Because the tumor originated from the left superior pulmonary vein and diffused into the left atrial wall and the junction of the right superior pulmonary vein and left atrium, the en bloc excision of the tumor was impossible. The first-line chemotherapy, including ifosfamide, doxorubicin, and dacarbazine, in conjugation with radiation therapy could not demonstrate any effect on the tumor size. However, the following PAX-containing regimen provided complete regression of the tumor. After PAXbased high-dose chemotherapy with autologous peripheral blood stem cell transplantation, the patient showed complete remission for 2 years. Although he suffered metastatic recurrences and died 4 years after the onset of symptoms, our patient's clinical course clearly reveals the efficacy of PAX.




\section{Case Reports in Oncology}

Kounami et al.: Efficacy of Paclitaxel in a Patient with Inoperable Pulmonary Vein Leiomyosarcoma

\section{Introduction}

Primary tumors of the great vessels are very rare, and most of the tumors are sarcomas. Among them, primary pulmonary vein leiomyosarcoma is extremely rare and has a poor prognosis $[1,2]$. Not much is known about the best therapeutic modality for primary pulmonary vein leiomyosarcoma. Surgical excision with a wide margin is the only mainstay of curative treatment, and the role of chemotherapy and radiation therapy is controversial [2]. Here, we report a case with pulmonary vein leiomyosarcoma, wherein the use of paclitaxel (PAX) proved to be effective.

\section{Case Presentation}

An 18-year-old male patient was hospitalized because of a 1-week history of cough, fever, and shortness of breath. Both past medical history and family history were unremarkable. He was diagnosed with pneumonia, but antibiotics did not improve his condition, and he needed oxygen inhalation for respiratory distress. One week later, a cardiovascular examination revealed 2/6 diastolic murmur over the apex and a transthoracic echocardiogram revealed a left atrial mass. He was emergently referred to our hospital. A chest radiograph revealed cardiomegaly and pulmonary vascular congestion. A transthoracic echocardiogram showed a large left atrial mass occluding the mitral inflow. A thoracic computed tomography presented a left atrial mass but no other tumors elsewhere (Fig. 1). Soon after admission, his condition rapidly deteriorated after massive hemoptysis to necessitate mechanical ventilation and percutaneous cardiopulmonary support. The patient underwent an emergent surgery under a putative diagnosis of left atrium myxoma. Median sternotomy, aortic-bicaval cannulation, and standard cardiopulmonary bypass were performed. Through a right interatrial groove incision, a large pedunculated hard mass occupying the left atrium was excised; the pedicle was traced to the left superior pulmonary vein. The cardiac surgeons indicated that the tumor might have originated from the left superior pulmonary vein near the left atrium and may have directly invaded the atrial wall to the junction of the right superior pulmonary vein and left atrium. Although the mass was removed as much as possible, the en bloc removal of the tumor, with both superior pulmonary veins and their corresponding pulmonary lobes and atrial wall, could not be performed.

Histologically, the tumor comprised pleomorphic cells with bizarre nuclei and some spindle cells with blunt-ended nuclei. In addition, multinucleated giant cells and occasional coagulative necrosis $(<50 \%$ in the examined area) were observed. Besides, mitotic figures were also found with a frequency of 5-7 per 10 high-power fields. The immunohistochemical staining with the proliferation marker Ki- 67 revealed a proliferation index of $50 \%$. While the tumor cells were $\alpha$-smooth muscle actin, h-caldesmon, desmin, and vimentin positive, epithelial membrane antigen, keratin, CD34, and factor VIII were negative (Fig. 2). Hence, the diagnosis of leiomyosarcoma was determined.

After the operation, he required the assistance of percutaneous cardiopulmonary support for 5 days and mechanical ventilation for 12 days. However, the remaining tumor grew rapidly during this interval. A transesophageal echocardiography revealed a mass, measuring $1 \times 1.5 \mathrm{~cm}$ in the left superior pulmonary vein 7 days after the operation, and the tumor grew up to occupying two-thirds of the left atrium at postoperative day 40 . Because the curative en bloc excision of the tumor was impracticable, chemotherapy and combined radia- 
tion therapy (total amount of $60 \mathrm{~Gy}$ ) were initiated at postoperative days 46 and 59, respectively.

One course of IFO/DOX: ifosfamide, $2.5 \mathrm{~g} / \mathrm{m}^{2} \times 5$ days, doxorubicin, $20 \mathrm{mg} / \mathrm{m}^{2} \times 2$ days; 2 courses of CYVADIC: cyclophosphamide, $1,200 \mathrm{mg} / \mathrm{m}^{2} \times 1$ day, vincristine, $1 \mathrm{mg} / \mathrm{m}^{2} \times 2$ days, doxorubicin, $40 \mathrm{mg} / \mathrm{m}^{2} \times 1$ day, dacarbazine, $225 \mathrm{mg} / \mathrm{m}^{2} \times 4$ days; 1 course of MAID: ifosfamide, $2.5 \mathrm{~g} / \mathrm{m}^{2} \times 3$ days with MESNA, doxorubicin, $20 \mathrm{mg} / \mathrm{m}^{2} \times 3$ days, dacarbazine, $225 \mathrm{mg} / \mathrm{m}^{2} \times 3$ days; and selective tumor-specific intra-arterial infusion of melphalan (50 $\mathrm{mg}$ ) via internal thoracic artery could not achieve any response. For salvage chemotherapy, a PAX-containing regimen, ifosfamide, $1 \mathrm{~g} / \mathrm{m}^{2} \times 3$ days, doxorubicin, $30 \mathrm{mg} / \mathrm{m}^{2} \times 1$ day, PAX, $140 \mathrm{mg} / \mathrm{m}^{2} \times 1$ day (IFO/DOX/PAX), was initiated, and it achieved dramatic tumor regression (Fig. 3). The tumor almost disappeared after 2 courses with IFO/DOX/PAX. After 4 courses of IFO/DOX/PAX, we conducted a tandem PAX-based high-dose chemotherapy (HDC) with autologous peripheral blood stem cell transplantation (PBSCT); the first HDC regimen comprised ifosfamide, $3 \mathrm{~g} / \mathrm{m}^{2} \times 3$ days and PAX, $370 \mathrm{mg} / \mathrm{m}^{2} \times 1$ day, and the second comprised etoposide, $300 \mathrm{mg} / \mathrm{m}^{2} \times 3$ days, carboplatin, $400 \mathrm{mg} / \mathrm{m}^{2} \times 3$ days, and PAX, 420 $\mathrm{mg} / \mathrm{m}^{2} \times 1$ day, respectively. The patient achieved complete regression of the tumor.

The patient enjoyed a sustained complete remission for 2 years after the therapy, but he suffered a brain metastasis followed by bone and pulmonary metastases. No local recurrence was noted. The lesions were refractory to chemotherapy, including PAX, and he underwent palliative surgery and radiation therapy. However, he died 4 years after the onset of symptoms and an autopsy was not allowed.

\section{Discussion}

Leiomyosarcoma of the pulmonary vein is very rare. Since its clinical presentation is nonspecific, early diagnosis is often difficult [2]. A magnetic resonance imaging and an 18Ffluorodeoxyglucose positron emission tomography are reportedly useful in examining the characteristics and extent of cardiac tumors [3]. In addition, the usefulness of preoperative biopsy has been also reported [4]. In our case, the aggressive clinical course did not allow precise preoperative examinations for the tumor. Because the mean age of cardiac sarcomas was reported to be fourth to fifth decades [1,2], a preoperative diagnosis of our patient was the left atrium myxoma.

Owing to the difficulty of determining the site of a large tumor intraoperatively, and as left atrial leiomyosarcoma often diffuses into the pulmonary vein lumen, in some previously reported cases, pulmonary vein leiomyosarcoma has been doubted to be misinterpreted as left atrial leiomyosarcoma [5, 6]. We diagnosed our patient with leiomyosarcoma derived from the left superior pulmonary vein near the left atrium from the findings at operation and the growth pattern of the remaining tumor after the operation; however, we could not demonstrate histologically because the en bloc removal of the tumor was impossible.

Including the present case, only 30 cases with leiomyosarcoma of the pulmonary veins have been reported to date [2,7-12]. Owing to the scarcity of this disease, little is known about the optimal treatment modality of pulmonary vein leiomyosarcoma. A radical surgical resection with a wide margin seems to offer the only chance of cure. Although successful simultaneous removal of the corresponding lung with extensive resection of the tumor and atrial wall en bloc has been reported [5], the results of surgical treatment have been palliative in most cases because of the tumor relapses or metastases $[2,10]$. While an adjuvant therapy is anticipated under these circumstances, the role of chemotherapy and radiation 


\section{Case Reports in Oncology}

Kounami et al.: Efficacy of Paclitaxel in a Patient with Inoperable Pulmonary Vein Leiomyosarcoma

therapy has not been elucidated. Concerning radiation therapy, leiomyosarcoma has been reported to show low radiosensitivity [2]. The benefits of chemotherapy are also unclear. In some cases, postoperative chemotherapy might prolong the survival $[8,10]$, but a possibility that the supposed efficacy of chemotherapy was coupled with surgical interventions should be taken into account because surgery alone might contribute to the prolongation of survival [5]. In addition to unknown efficacy, there have been no data on an optimal chemotherapy regimen. Doxorubicin, ifosfamide, etoposide, dacarbazine, vincristine, and cyclophosphamide have been used as in other soft tissue sarcomas, and there was no observed exact efficacy of chemotherapy $[2,8,10]$.

Our case clearly exhibits the efficacy of PAX. Initially, our patient underwent IFO/DOX followed by CYVADIC and MAID, in conjunction with radiation therapy, but these could not demonstrate any effect on the tumor size. Nevertheless, PAX-containing chemotherapy regimen resulted in the disappearance of the tumor and sustained complete remission for 2 years. Whether PAX only or its combination with ifosfamide and doxorubicin was effective could not be determined in our case. Generally, PAX is used in third-line chemotherapy in the treatment soft tissue sarcomas [13]. The use of PAX has been described only in 2 cases with pulmonary vein leiomyosarcoma for salvage chemotherapy, but with no efficacy [10,14]. Although a further experience of PAX in cardiac leiomyosarcoma is needed, the clinical course of our patient indicated that PAX-containing chemotherapy, IFO/DOX/PAX, might be a candidate for the first-line chemotherapy for pulmonary vein leiomyosarcoma.

Since our patient's tumor had been highly sensitive to PAX, we conducted a tandem PAX-based HDC with PBSCT [15]. This is the first report of a patient with pulmonary vein leiomyosarcoma who underwent HDC with autologous PBSCT. He achieved sustained complete remission for 2 years but suffered multiple metastatic recurrences. A further refinement of the treatment strategy, including molecularly targeted agents, is needed [13].

\section{Acknowledgement}

The authors would like to thank Enago (www.enago.jp) for the English language review.

\section{Statement of Ethics}

The authors have no ethical conflicts to disclose. Patient consent was graciously obtained for the publication needs.

\section{Disclosure Statement}

The authors declare that there is no conflict of interest regarding the publication of this paper.

\section{References}

1 Burke AP, Virmami R: Sarcoma of the great vessels. A clinicopathologic study. Cancer 1993;71:17611773. 


\section{Case Reports in Oncology}

\begin{tabular}{l|l}
\hline Case Rep Oncol 2017;10:564-570 \\
\hline DOI: 10.1159/000477657 & $\begin{array}{l}\text { C 2017 The Author(s). Published by S. Karger AG, Basel } \\
\text { www.karger.com/cro }\end{array}$ \\
\hline
\end{tabular}

Kounami et al.: Efficacy of Paclitaxel in a Patient with Inoperable Pulmonary Vein Leiomyosarcoma

-2 Hong SP, Choi JY, Son JY, Lee YS, Kim KS: Leiomyosarcoma of the pulmonary veins extending into the left atrium. Herz 2014;39:593-597.

-3 Tao TY, Yahyavi-Firoua-Abadi N, Singh GK, Bhalla S: Pediatric cardiac tumors: clinical and imaging features. Radiographics 2014;34:1031-1046.

-4 Davis GK, Jones EL, Bonser RS, Roberts DH: Coronary arteriographic and pathological findings in a case of primary leiomyosarcoma of the heart. Int J Cardiol 1997;23:313-316.

-5 Shimono T, Yuasa H, Yuasa U, Yasuda F, Adachi K, Tokui T, ais H, Takao M, Namikawa S, Yada I: Pulmonary leiomyosarcoma extending into left atrium or pulmonary trunk: complete resection with cardiopulmonary bypass. J Thorac Cardiovasc Surg 1998;115:460-461.

-6 Babatasi G, Massetti M, Galateau F, Khayat A: Leiomyosarcoma of the pulmonary veins extending into the left atrium or left atrial leiomyosarcoma: multimodality therapy. J Thorac Cardiovasc Surg 1998;115:665-666.

7 Donsbeck AV, Ranchere D, Coindre JM, Gall FL, Cordier JF, Loire R: Primary cardiac sarcomas: an immunohistochemical and grading study with long-term follow-up of 24 cases. Histopathology 1999;34:295-304.

-8 Gukop P, Frassetto G, Karapanagiotidis G, Chandrasekaran V: Leiomyosarcoma of pulmonary vein presenting as left atrial mass: an outline of management principles. Case Rep Cardiol 2012;2012:396319.

9 Patel SM, Kadakia KC, Maleszewski JJ, Marks RS: Straight to the heart: pulmonary vein leiomyosarcoma. Am J Med 2013;126:117-119.

10 Galeone A, Validire P, Debrosse D, Folliguet T, Laborde F: Leiomyosarcoma of the right inferior pulmonary vein: 2 years survival with multimodality therapy. Gen Thorac Cardiavasc Surg 2013;61:534-537.

11 Srividya VV, Sailendra V: An extremely rare case of left atrium and right pulmonary vein leiomyosarcoma. J Clin Diagn Res 2015;9:ED18020.

-12 Blachman-Braun R, Aboitiz-Rivera CM, Aranda-Fraustro A, Ransom-Rodriguez A, Baltazares-Lipp ME, Catrip-Torres JM, Martinez-Reding JO: Immunohistochemical diagnosis of primary cardiac leiomyosarcoma in a Latin American patient. Rare Tumors 2017;9:6669.

13 Ryan CW, Desai J: The past, present, and future of cytotoxic chemotherapy and pathway-directed targeted agents for soft tissue sarcoma. Am Soc Clin Oncol Educ Book DOI 10.1200/EdBook_AM.2013.33.e386.

14 Garraud M, Margery J, Laborde F, Saint-Blancard P, David S, Bonardel G, Héno P: Pulmonary vein leiomyosarcoma extending into the atrium. Rev Pneumol Clin 2010;66:321-325.

-15 Pal SK, Yamzon J, Sun V, Carmichael C, Saikia J, Ferrell B, Frankel P, Hsu J, Twardowski P, Stein CA, Margolin K: Paclitaxel-based high-dose chemotherapy with autologous stem cell rescue for relapsed germ cell tumor: clinical outcome and quality of life in long-term survivors. Clin Genitourin Cancer 2013;11:121-127.
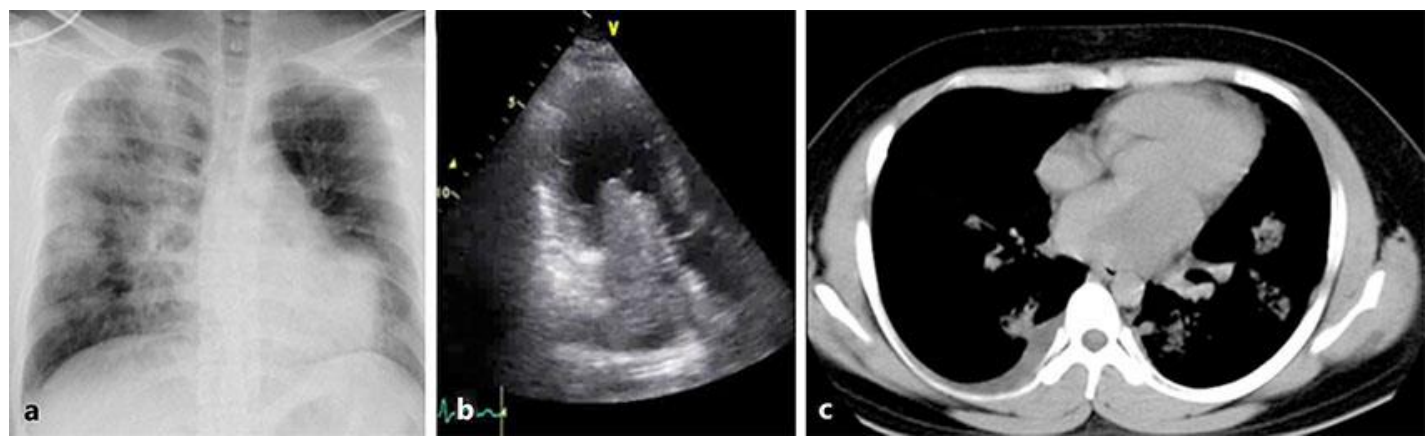

Fig. 1. Imaging studies at admission to our hospital. a Chest roentgenography showing mild cardiomegaly and severe pulmonary congestion. $\mathbf{b}$ Transthoracic echocardiogram showing the left atrial mass obstructing the mitral flow. c Plain thoracic computed tomography showing the left atrial mass. 


\section{Case Reports in Oncology}

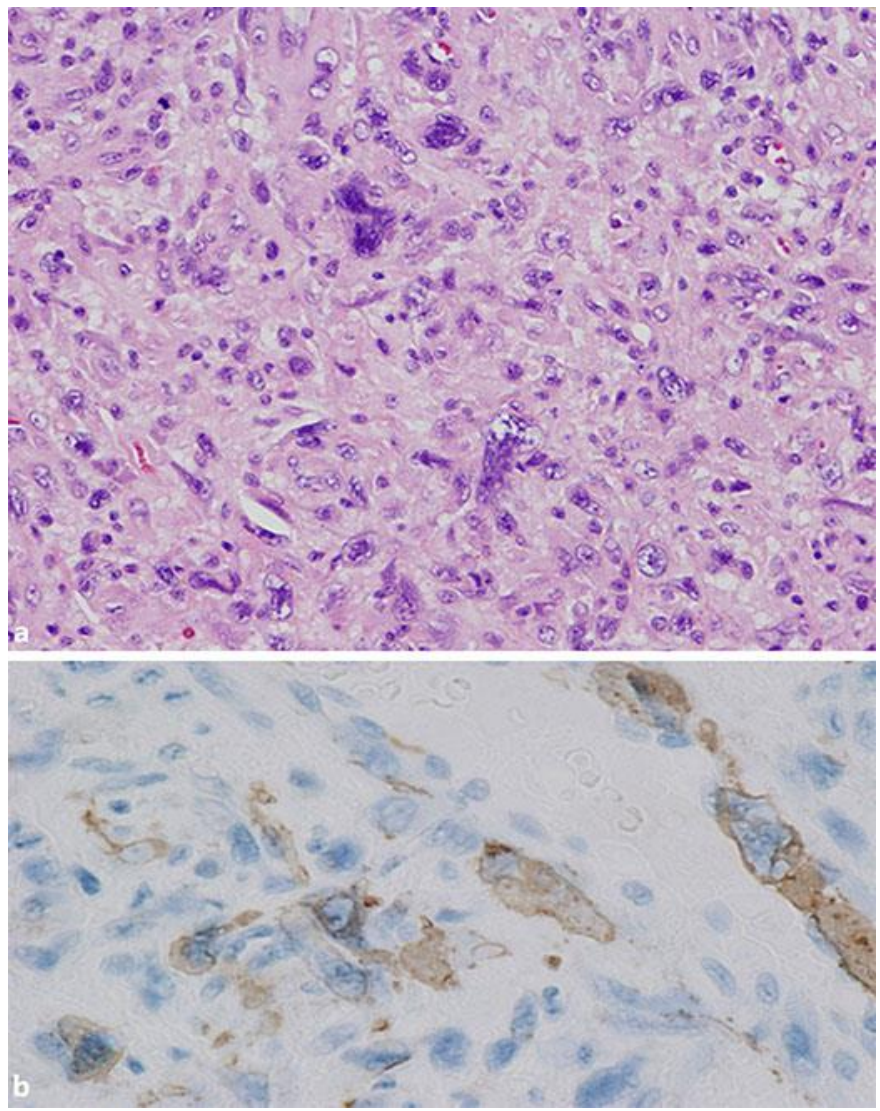

Fig. 2. Microscopic findings of the tumor. a Hematoxylin and eosin-stained section showed pleomorphic tumors with bizarre nuclei. b Immunohistochemical analysis showing $\alpha$-smooth muscle actin-positive tumor cells. 


\section{Case Reports in Oncology}
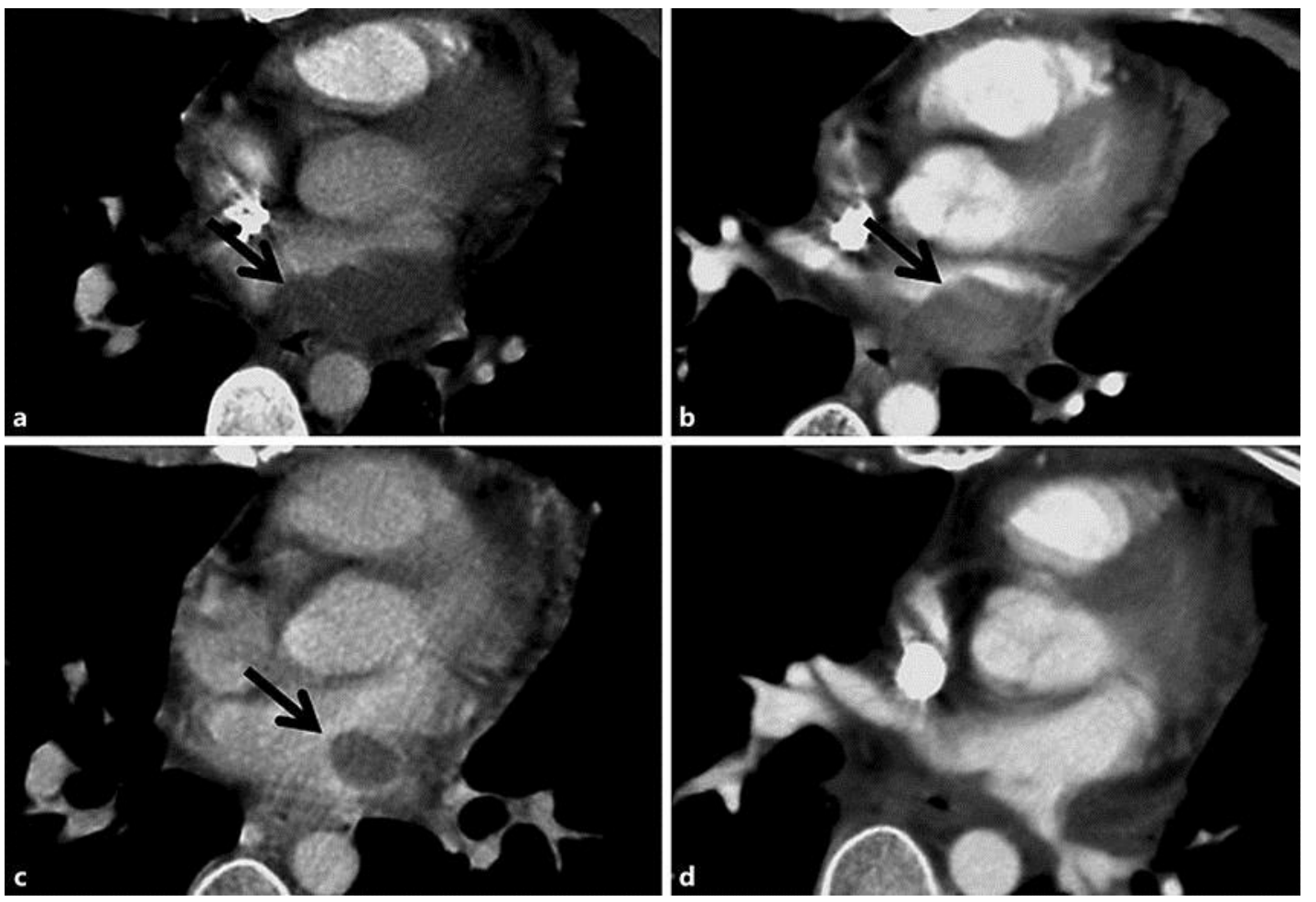

Fig. 3. Serial enhanced thoracic computed tomography during chemotherapy. a Before starting the chemotherapy. b After IFO/DOX, CYVADIC, MAID, and radiotherapy. The tumor size did not change. c After 1 course of IFO/DOX/PAX, the tumor markedly regressed. $\mathbf{d}$ Before first HDC, the tumor almost disappeared. 